\title{
A new species of Diphya Nicolet 1849 and the first description of the female of D. tanasevitchi from northern China (Araneae: Tetragnathidae: Metinae)
}

\author{
Hongmei $\mathrm{Yu}^{1}$, Feng Zhang ${ }^{1 *} \&$ Mikhail M. Omelko ${ }^{2,3}$ \\ ${ }^{I}$ The Key Laboratory of Invertebrate Systematics and Application, Hebei University, Baoding, Hebei 071002, China \\ ${ }^{2}$ Gornotayozhnaya Station FEB RAS, Gornotayozhnoye Vil., Ussuriyski Dist., Primorski Krai, 692533 Russia. \\ E-mail: omelkom@gmail.com \\ ${ }^{3}$ Far Eastern Federal University, Sukhanova 8, Vladivostok 690950, Russia. \\ *Corresponding author. E-mail: dudu06042001@163.com
}

\begin{abstract}
A new species, Diphya wulingensis, is described from Wuling Mountains, Hebei Province of China, and the female of D. tanasevitchi (Zhang, Zhang \& Yu 2003) is described and illustrated for the first time using the specimens from Liupan Mountains of Ningxia, and Wuling Mountains of Hebei.
\end{abstract}

Key words - spider, taxonomy, new discovery

\section{Introduction}

The genus Diphya was established by Nicolet in 1849 for D. macrophthalma Nicolet 1849 from Chile. Up to now, it includes 12 species which are distributed in East Asia, South America and Africa (Platnick 2013). Among them, five species are reported from China, D. okumae Tanikawa 1995, D. taiwanica Tanikawa 1995, D. qianica Zhu, Song \& Zhang 2003, D. tanasevitchi Zhang, Zhang \& Yu 2003 and D. songi Wu \& Yang 2010 (Platnick 2013).

Recently, while examining the specimens collected from Hebei and Ningxia Provinces of China, we found a new Diphya species and previously unknown female of $D$. tanasevitchi. Goal of this paper is to provide description of the new species and the female of D. tanasevitchi.

\section{Material and methods}

All measurements given in the text are in millimeters. Measurements of legs are given as follows: total length (femur + patella + tibia + metatarsus + tarsus). Epigyne was cleared in warm solution of $\mathrm{KOH}$, then it was transferred to alcohol and temporarily mounted for drawing. Epigyne and palpal organ were examined and drawn under a Leica M165C stereomicroscope equipped with an Abbe drawing device. Photographs were taken using a Leica M205A stereomicroscope. All specimens examined in this study are deposited in the Museum of Hebei University, Baoding, China (MHBU).

The following abbreviations are used: ALE, anterior lateral eye; AME, anterior median eye; AME-ALE, distance between AME and ALE; AME-AME, distance between AME; MOA, median ocular area; PLE, posterior lateral eye; PME, posterior median eye; PME-PLE, distance between PME and PLE; PME-PME, distance between PME.

\section{Taxonomy}

Diphya Nicolet 1849

Diphya wulingensis sp. nov.

(Figs. 1-13)

Types. Holotype $\delta$, China, Hebei Province, Xinglong County, Wuling Mountains, 13 August 2009, Mingsheng Zhu leg. Paratypes: 5 우우, same data as for holotype; 18우우, $5 \overbrace{}^{\lambda}$, same locality, 12 August 2009, Mingsheng Zhu leg.

Diagnosis. The new species is similar to D. okumae (Zhu et al. 2003: 56, fig. 22A-I) by the general appearance and structure of the palpal organ, but can be distinguished from it by: 1) thick, short and obtuse embolus (Figs. 5-7, 10-12) (whip-shaped embolus in the latter);2) the triangular conductor (Figs. 6, 11) (rectangular in the latter); 3) epigynal plate with a pair of semicircular depressions (Figs. $3-4,8^{-9}$ ) (oval depression in the latter).

Etymology. The specific name refers to the type locality.

Description. Male (holotype). Total body length 2.60; carapace length 1.28 , width 1.07 ; abdomen length 1.53 , width 1.02. Carapace (Fig. 1) pyriform, the anterior part black brown and the posterior part orange-yellow, lateral margins with two dark longitudinal stripes (other male specimens almost same color and pattern). Cephalic region raised conspicuously. Cervical groove and radial grooves conspicuous. Fovea pit-shaped. Clypeus height 0.15. Both eye rows recurved (in dorsal view), with dark brown ring around eye, indirect eyes without tapetum. Eye sizes and interdistances: AME 0.05, ALE 0.13, PME 0.13, PLE 0.15; AME-AME 0.05, AME-ALE 0.05, PME-PME 0.05, PMEPLE 0.025. MOA trapezoidal, wider than long (0.35: 0.30), narrower in front than in back $(0.15: 0.30)$. Chelicerae 


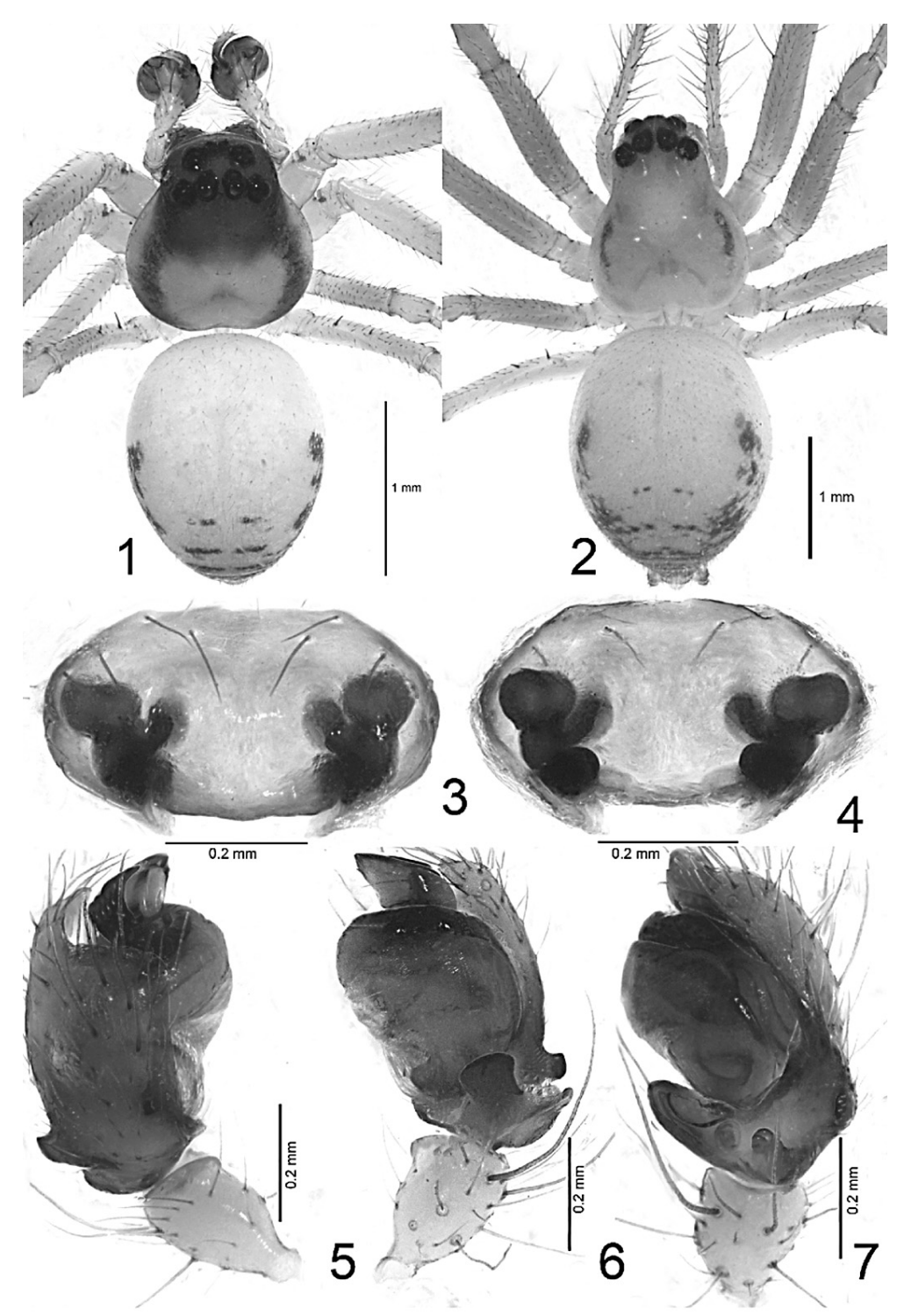

Figs. 1-7. Diphya wulingensis sp. nov. 1, male body, dorsal view; 2, female body, dorsal view; 3, epigyne, ventral view; 4, same, dorsal view; 5, left male palp, prolateral view; 6, same, ventral view; 7, same, retrolateral view. Scales: $1 \mathrm{~mm}(1-2) ; 0.2 \mathrm{~mm}(3-7)$.

brown, with 3 promarginal and 4 retromarginal teeth. Sternum triangular, yellowish brown. Legs orange, metatarsi and tibiae I, II prolaterally with a row of thin spines. Leg measurements: I $6.23(1.50,0.50,1.70,1.88,0.65)$; II $5.61(1.50,0.38,1.48,1.75,0.50)$; III $3.23(0.88,0.35,0.75$, $0.75,0.50)$; IV $4.22(1.23,0.28,1.05,1.08,0.58)$. Abdomen (Fig. 1) light orange, with black marks on both sides (some male specimens with unconspicuous black marks). Palpal femur slightly longer than tibia, patella shortest; paracymbium with four apophyses (Figs. 7, 13); bulb with spherical tegulum; embolus sickle-shaped, with broad base, thick and obtuse tip (Figs. 10, 12); conductor triangular, weakly sclerotized (Figs. 5-7).

Female. Total length 3.21-3.57. One female with body length 3.57: carapace length 1.53, width 1.28; abdomen length 2.14, width 1.79. Carapace (Fig. 2) pyriform, orange-yellow, the anterior part edge with two light brown color lengthways stripes, other specimens without too much change. Cephalic region raised conspicuously. Cervical groove and radial grooves conspicuous. Clypeus height 0.20 . Indirect eyes without tapetum. Eye sizes and interdistances: AME 0.05, ALE 0.13, PME 0.13, PLE 0.15; AME-AME 0.03, AME-ALE 0.05, PME-PME 0.08, PMEPLE 0.05; MOA trapezoidal, wider than long (0.43: 0.38), narrower in front than in back $(0.18: 0.38)$. Chelicerae brown, with 3 promarginal and 4 retromarginal teeth. Sternum triangular, yellowish brown. Leg measurements: I $6.69(1.63,0.63,1.63,2.00,0.80)$; II $6.02(1.50,0.63,1.38$, $1.78,0.73)$; III $3.67(1.00,0.38,0.83,0.88,0.58)$; IV 4.61 $(1.13,0.38,1.20,1.25,0.65)$. Other characters as in male. Epigynal plate with pair of semicircular depressions, spermathecae spherical (Figs. 3-4, 8-9), and spermathecae with many "hairs" (glandular structures, which unknown in other Araneoidea) inner sides (Fig. 9). 

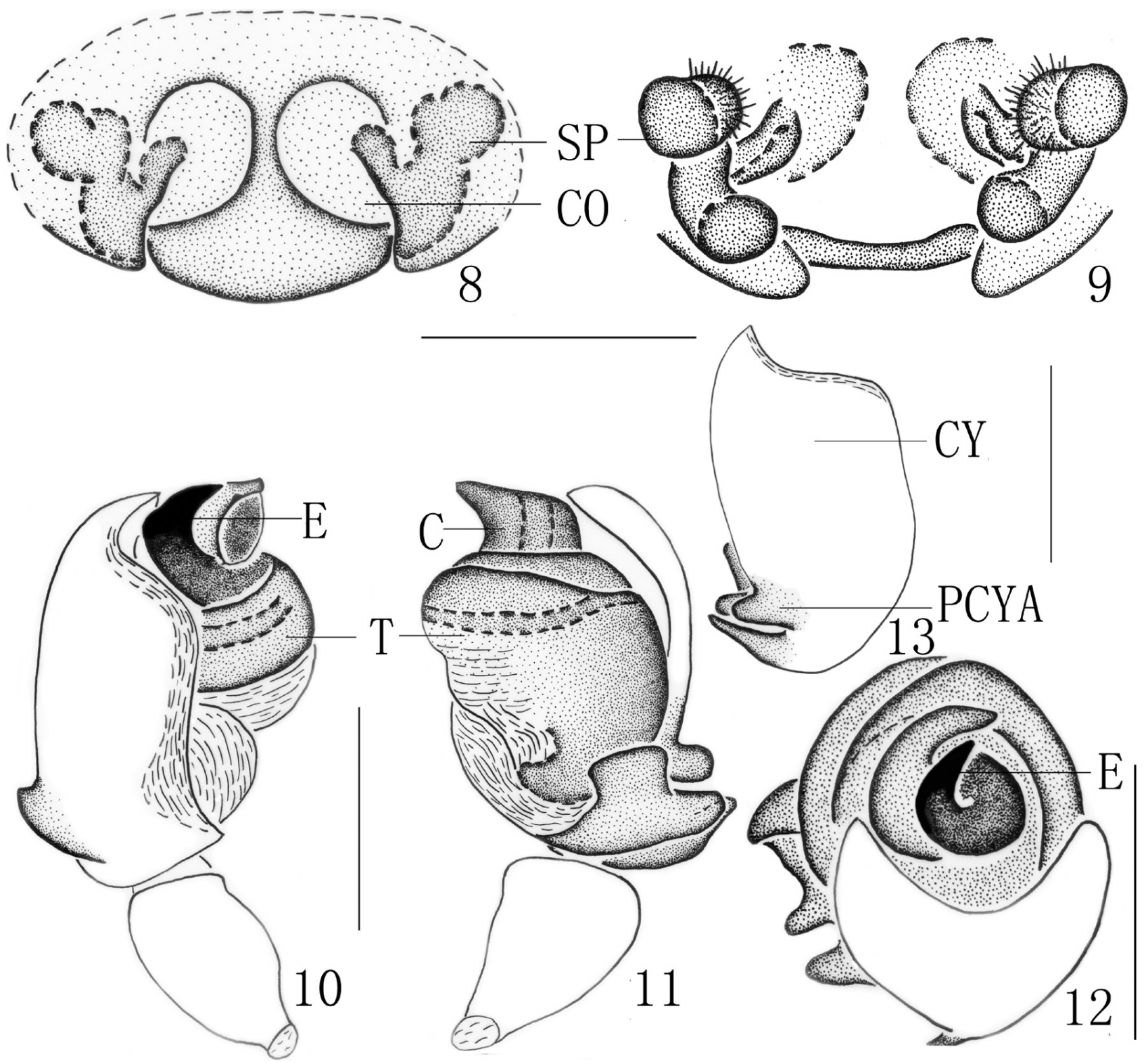

Figs. 8-13. Diphya wulingensis sp. nov. 8, epigyne, ventral view; 9, same, dorsal view, showing special hairs; 10, left male palp, prolateral view; 11, same, retrolateral view; 12, same, apical view; 13, same, dorsal view. Scales: 0.2 mm. Abbreviations: C, conductor; CO, copulatory openings; CY, cymbium; E, embolus; PCYA, paracymbium apophysis; SP, spermatheca; T, tegulum.

Distribution. China: Hebei.

Diphya tanasevitchi (Zhang, Zhang \& Yu 2003)

(Figs. 14-25)

Lophomma tanasevitchi Zhang et al. 2003: 407, f. 1A-C (ㄱ). Diphya tanasevitchi: Marusik et al. 2007: 163.

Material examined. CHINA: Ningxia Hui Autonomous Region, Liupanshan National Natural Reserve: 1万, 2우우, Jingyuan County, Longtan Forest Farm, 21 June 2008, Chao Zhang leg.; 1우, Xixia Forest Farm, 27 June 2008, Chao Zhang leg.; 1우, Longde County, Sutai Forest Farm, 2 July 2008, Chao Zhang leg.; Hebei Province: 1우, Wulingshan
National Natural Reserve, Xinglong County, Shangshidong Town, 30 July 2012, Long Liu leg.

Diagnosis of female. Female of this species is similar to that of $D$. qianica (Zhu et al. 2003: 57, fig. 23A-C), both have a septum, and the anterior part of septum narrow, the middle part of septum wide, but can be distinguished from it by: 1) general appearance and structure of the spermathecae simple, kidney-shaped (Figs. 16-17, 21-22) (folded and bent in D. qianica), both sides of abdomen black brown (Figs. 15) (light brown in D. qianica).

Description. Male. Body length 2.30; carapace length 1.05 , width 1.00 ; abdomen length 1.20 , width 1.03 . Carapace (Fig. 14) oval, brown. Cephalic region raised conspicuously. Cervical groove and radial grooves 

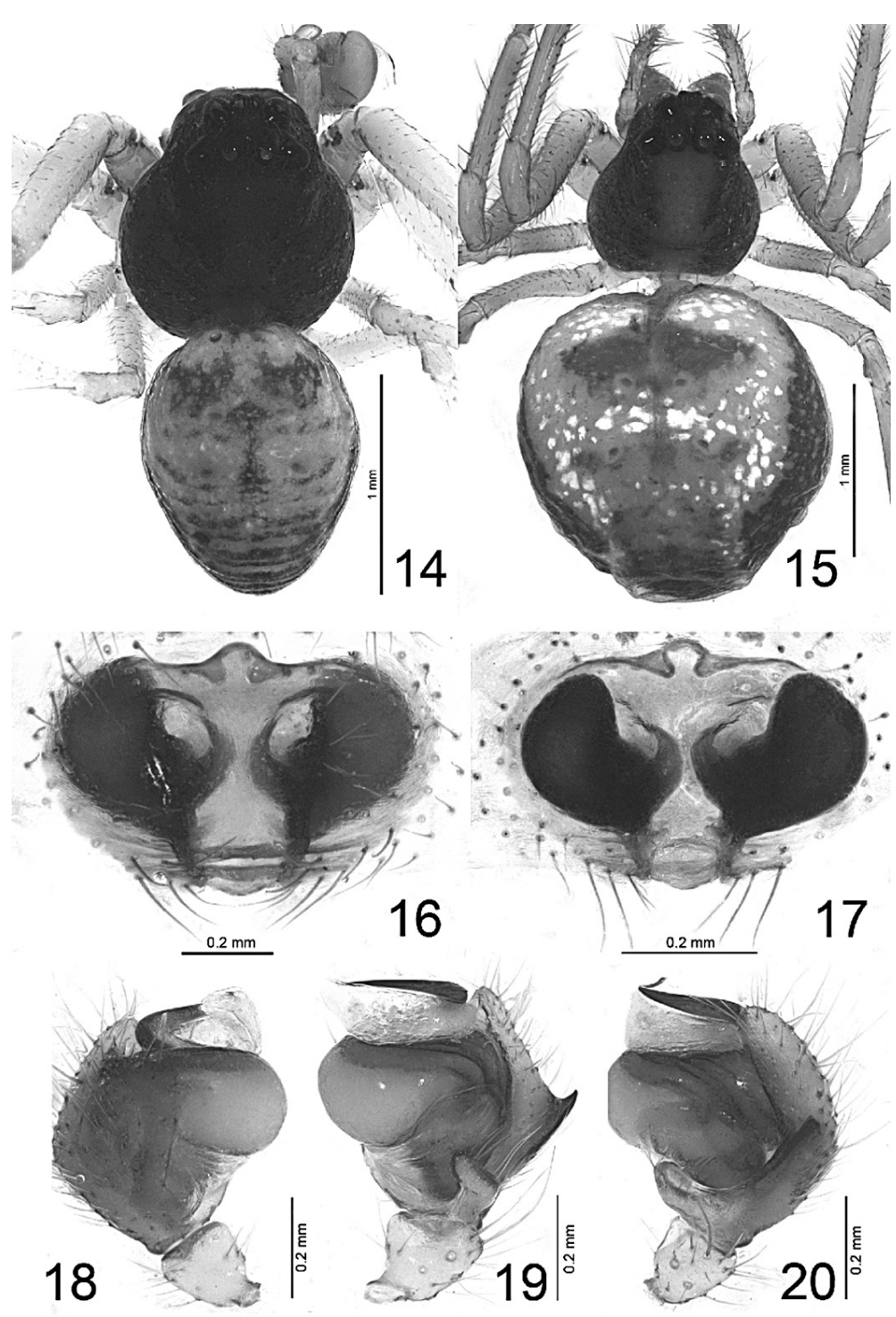

Figs. 14-20. Diphya tanasevitchi. 14, male body, dorsal view; 15, female body, dorsal view; 16, epigyne, ventral view; 17 , same, dorsal view; 18, male left palp, prolateral view; 19, same, ventral view; 20, same, retrolateral view. Scales: $1 \mathrm{~mm}(14-15)$; 0.2 mm (16-20).

conspicuous. Fovea pit-like. Both eye rows recurved (in dorsal view), indirect eyes without tapetum. Clypeus height 0.20. Eye sizes and interdistances: AME 0.03, ALE 0.08, PME 0.08, PLE 0.08; AME-AME 0.03, AME-ALE 0.05, PME-PME 0.08, PME-PLE 0.10. MOA trapezoidal, longer than wide $(0.25: 0.20)$, narrower in front than in back $(0.15$ : 0.25). Chelicerae brown, with 3 promarginal and 4 retromarginal teeth. Sternum triangualr, yellowish brown. Legs orange, metatarsi and tibiae I, II prolaterally with a row of thin spines. Leg measurements: I 4.42 (1.13, 0.38, $1.13,1.13,0.65)$; II $3.71(0.95,0.35,0.93,0.88,0.60)$; III $2.06(0.70,0.20,0.48,0.43,0.25)$; IV $2.87(0.88,0.25,0.68$, $0.68,0.38)$. Abdomen oval, longer than wide, the anterior part round, while the posterior part sharp; gray dorsally, with dark brown stripes on both sides; the middle part with two pairs of obvious muscle impressions, the anterior one pair black spot, the posterior one pair dumb-bell-shaped spots. The apophysis of cymbium swallow-tailed, tegulum big, embolus whip-shaped, bent slightly; conductor semicircular, translucent (Figs. 18-20, 23-25).

Female. Total length 3.14-3.94. One female with body length 3.14: carapace length 1.15, width 1.00; abdomen length 1.88, width 1.75. Carapace (Fig. 15) yellowish brown, both sides color deeper than the middle part of it, other specimens without too much change. Clypeal height 0.13. Eye sizes and interdistances: AME 0.03, ALE 0.13, PME 0.13, PLE 0.13; AME-AME 0.03, AME-ALE 0.05, PME-PME 0.08, PME-PLE 0.10; MOA trapezoidal, longer than wide $(0.28: 0.23)$, narrower in front than in back $(0.13$ : 0.28). Chelicerae brown, with 3 promarginal and 4 retromarginal teeth. Leg measurements: I 4.18 (1.05, 0.40, $1.13,0.95,0.65)$; II $4.06(1.08,0.38,1.00,1.00,0.60)$; III $2.38(0.80,0.20,0.50,0.50,0.38)$; IV 3.34 (1.13, 0.25, 0.75, $0.78,0.43)$. Abdomen almost equal between long and wide, longer than wide, both sides and the middle part of it with the silver spots. Other characters as in male. Anterior 

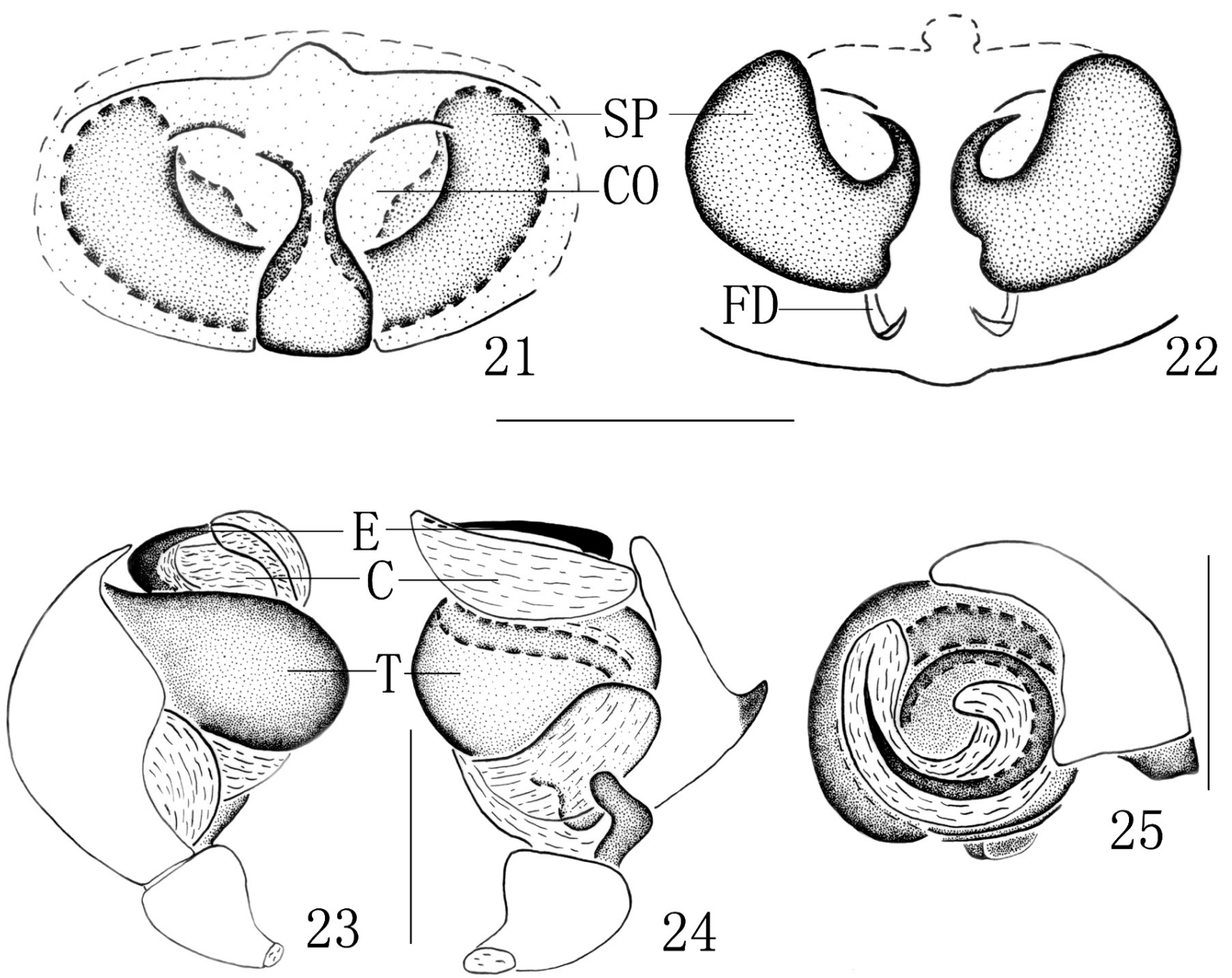

Figs. 21-25. Diphya tanasevitchi. 21, epigyne, ventral view; 22, same, dorsal view; 23, left male palp, prolateral view; 24, same, retrolateral view; 25 , same, apical view. Scales: $0.2 \mathrm{~mm}$. Abbreviations: C, conductor; CO, copulatory openings; E, embolus; FD, fertilization duct; SP, spermatheca; T, tegulum.

margin of epigyne strongly sclerotized. Anterior part of septum narrow, the middle part of septum wide. Spermathecae kidney-shaped (Figs. 16-17, 21-22).

Distribution. China: Hebei, Ningxia (it is the westernmost species of the genus yet found in Asia)

\section{Acknowledgments}

We are grateful to late Prof. Mingsheng Zhu and Dr. Chao Zhang for collecting the specimens. This work was supported by the National Natural Science Foundation of China (No. 31372154, 31093430) and in part by a Program of Ministry of Science and Technology of the Republic of China (2012FY110803) to Dr. Feng Zhang.

\section{References}

Marusik, Y. M., Gnelitsa, V. A. \& Koponen, S. 2007. A survey of Holarctic Linyphiidae (Aranei). 4. A review of the erigonine genus Lophomma Menge, 1868. Arthropoda Selecta, 15: 153-171.
Platnick, N. I. 2013. The world spider catalog, version 14.0. American Museum of Natural History, New York. Available from: http://research.amnh.org/entomology/spiders/catalog/index.html (accessed 22 October 2013)

Tanikawa, A. 1995. Two new species of the spider genus Diphya (Araneae: Tetragnathidae) from Japan and Taiwan, with notes on the known species. Acta Arachnol., 44: 101-111.

Wu, A. G. \& Yang, Z. Z. 2010. Description of a new species of the genus Diphya from Cangshan National Nature Reserve of Yunnan province, China (Araneae, Tetragnathidae). Acta Zootaxon. Sinica, 35: 594-596.

Zhang, F., Zhang, Y. X. \& Yu, H. D. 2003. One new record genus and two new species of the family Linyphiidae (Arachnida: Araneae) from China. J. Hebei Univ., 23: 407-410.

Zhu, M. S., Song, D. X. \& Zhang, J. X. 2003. Fauna Sinica: Invertebrata Vol. 35: Arachnida: Araneae: Tetragnathidae. Science Press, Beijing, 418 pp.

Received December 8, 2013 / Accepted May 15, 2014 